\title{
The SBCCP and its recommendations: consensus or dissent?
}

\author{
Antonio José Gonçalves ${ }^{1}$ \\ How to cite: Gonçalves AJ. The SBCCP and its recommendations: consensus or dissent?. \\ Arch Head Neck Surg. 2021;50:e202150128. https://doi.org/10.4322/ahns.2021.0009
}

São Paulo, 10 August 2021

Nowadays, Specialty Societies (SS) are the foothold of medical development. They hold technical knowledge about matters related to their coverage area, dictate conducts, provide the professional defense of physicians, organize scientific events, regulate contests to qualify new specialists, and promote social activities; in short, they play a fundamental role in the lives of physicians.

Another important aspect of the activity of the SS is the preparation of consensuses and guidelines for diagnoses and conducts in the most varied topics of their specialties. The Brazilian Society of Head and Neck Surgery (SBCCP) has been doing this routinely whenever there is new knowledge that requires this demand.

The SBCCP has recently done this with three new demands: robotic surgery, radiofrequency ablation of thyroid nodules, and transoral endoscopic thyroidectomy via a vestibular approach. At the forum of new technologies, these three techniques were discussed and a document was prepared and made available for consultation for 30 days aiming to gather opinions from all SBCCP members who wished to contribute to the improvement of the recommendations.

Robotic surgery is already well defined in the transoral approach and has been gaining adherents to cervical approaches, especially for neck dissections. The Brazilian Federal Council of Medicine (CFM) has issued an opinion $(041 / 2017)$ stating that robotic thyroidectomy is an experimental procedure. The SBCCP recommends that physicians and services use the robotic route for thyroidectomy under strict research protocols, with a well-documented Informed Consent Form (ICF) and in an absolutely transparent way, so that

Copyright Gonçalves This is an Open Access article distributed under the terms of the Creative Commons Attribution License, which permits unrestricted use, distribution, and reproduction in any medium, provided the original work is properly cited. ethics is preserved and the decision is shared between physicians and patients.

There was a consensus that radiofrequency ablation of thyroid nodules is a good method for selected cases and, at first, for benign cases, especially for patients with high surgical risk or who do not wish to undergo thyroidectomy. 
No consensus was reached regarding endoscopic thyroidectomy. It was clear that its benefit would be to avoid scarring and that it would be indicated in selected cases performed by properly trained surgeons.

The fact is that there was excessive advertising of this method by some physicians through social media, but especially by sensationalistic journalists or lay people. This advertising presents a real risk of commodifying this method, and the SBCCP does not agree with this.

There is no doubt that the main controversy among our peers is not the new surgical approach (the SBCCP and its associates have always favored the discussion of new techniques and technologies), but mainly its inadequate dissemination. Even because some of our training services already offer courses on this technique and perform the procedure in selected cases and with trained surgeons.

The SBCCP recommends that physicians and services that use this access route avoid advertising, especially on social networks, and discuss this approach with their patients in their offices, applying an ICF ethically and privately, as it should be done in all cases. The SBCCP will make ICFs (for open thyroidectomy and transoral endoscopic thyroidectomy) available in the restricted area of its website for the members who wish to use them.

Thus, the SBCCP will publish recommendations on radiofrequency ablation and robotic surgery.

In fact, bringing the robotics certification to our Society was a great victory. As for endoscopic thyroidectomy, we are proposing a national research, led by the SBCCP Department of Epidemiology, which will analyze the results of a prospective, non-randomized study carried out in our country, under our conditions, by surgeons with experience in the method, for selected cases. This study is aimed at producing well-grounded conclusions to better guide our recommendation.

Regarding the experimental nature of this surgery, we are convinced that a statement on this issue should be an exclusive attribution of the CFM, and that neither the SS, nor any other institution can be responsible for this.

I would like to end by answering the title question of this editorial: Consensus or dissent? In our view, there was a descent, and that is why this issue needs maturing.

A consensus is not always possible in Medicine. We have heard the phrase "Medicine is the science of transitory truths" a few times.

It is essential that we discuss at a high level, with respect to contradiction, without passion, but with prevailing common sense and with the best science available, and that we advise our associates on what is most appropriate, even if it is not unanimous, in order to ultimately achieve our final goal: to offer the best to our patients, which is the main purpose of our profession.

We strongly believe that we have fulfilled our mission at the SBCCP. It was a great pleasure to work with this Board. We hope we have left a legacy. Rest assured that this has been our desideratum since the first day of our administration. 
We are grateful to all SBCCP members for their support to our success as well as for their criticism to our mistakes. The last event organized by this administration will be our Brazilian Congress - September 2nd to 4th. We are certain that, despite being virtual, it will be one of the best scientific meetings of the SBCCP.

I am convinced that, despite occasional disagreements, we will remain united, always willing to listen to all our associates. I am leaving the presidency, but will remain in the Board of Directors, helping our Society in the best possible way. Our goal has always been and will continue to be the continuous development of our specialty and of the SBCCP. 